\title{
The Postnatal Accumulation of Junctional E-Cadherin Is Inversely Correlated with the Capacity for Supporting Cells to Convert Directly into Sensory Hair Cells in Mammalian Balance Organs
}

\author{
Maria Sol Collado, Benjamin R. Thiede, Wendy Baker, Charles Askew, Lisa M. Igbani, and Jeffrey T. Corwin \\ Department of Neuroscience, University of Virginia School of Medicine, Charlottesville, Virginia 22908
}

\begin{abstract}
Mammals experience permanent impairments from hair cell $(\mathrm{HC})$ losses, but birds and other non-mammals quickly recover hearing and balance senses after supporting cells (SCs) give rise to replacement HCs. Avian HC epithelia express little or no E-cadherin, and differences in the thickness of F-actin belts at SC junctions strongly correlate with different species' capacities for HC replacement, so we investigated junctional cadherins in human and murine ears. We found strong E-cadherin expression at SC-SC junctions that increases more than sixfold postnatally in mice. When we cultured utricles from young mice with $\gamma$-secretase inhibitors (GSIs), striolar SCs completely internalized their E-cadherin, without affecting N-cadherin. Hes and Hey expression also decreased and the SCs began to express Atoh1. After $48 \mathrm{~h}$, those SCs expressed myosins VI and VIIA, and by $72 \mathrm{~h}$, they developed hair bundles. However, some scattered striolar SCs retained E-cadherin and the SC phenotype. In extrastriolar regions, the vast majority of SCs also retained E-cadherin and failed to convert into HCs even after long GSI treatments. Microscopic measurements revealed that the junctions between extrastriolar SCs were more developed than those between striolar SCs. In GSI-treated utricles as old as P12, differentiated striolar SCs converted into HCs, but such responses declined with age and ceased by P16. Thus, temporal and spatial differences in postnatal SC-to-HC phenotype conversion capacity are linked to the structural attributes of E-cadherin containing SC junctions in mammals, which differ substantially from their counterparts in non-mammalian vertebrates that readily recover from hearing and balance deficits through hair cell regeneration.
\end{abstract}

\section{Introduction}

Debilitating hearing and balance deficits commonly arise when hair cells (HCs) are killed by loud sound, infections, or toxicity or die during aging. For humans and other mammals, HC deficits are permanent, but in fish, amphibians, and birds, supporting cells (SCs) can give rise to replacement HCs that restore sensory function. In seeking to identify differences that may limit regeneration in mammalian ears, we found that F-actin belts at apical SC-SC junctions grow exceptionally thick as balance organs mature in the first weeks after birth (Burns et al., 2008). That growth is inversely correlated $(r=-0.98)$ with measured declines in the propensity for SCs to change shape and proliferate after epithelium injury (Davies et al., 2007; Gu et al., 2007; Meyers and Corwin, 2007; Burns et al., 2008; Lu and Corwin, 2008). Comparable F-actin belts in SCs of fish, amphibians, and birds stay thin

Received May 20, 2011; revised June 20, 2011; accepted June 23, 2011.

Author contributions: M.S.C. and J.T.C. designed research; M.S.C., B.R.T., W.B., C.A., and L.M.I. performed research; M.S.C. contributed unpublished reagents/analytic tools; M.S.C., B.R.T., W.B., and L.M.I. analyzed data; M.S.C. and J.T.C. wrote the paper.

This work was supported by NIDCD/NIH Grant R01 DC000200 and by funds from the Lions of Virginia Hearing Research Foundation (J.T.C.). We thank Joseph Burns for helpful discussions and Dr. George Hashisaki for providing human inner ear specimens.

Correspondence should be addressed to Dr. Maria Sol Collado, Department of Neuroscience, University of Virginia Medical School, P.0. Box 801392, 409 Lane Road, Charlottesville, VA 22908. E-mail: mc4sr@virginia.edu.

DOI:10.1523/JNEUROSCI.2525-11.2011

Copyright $\odot 2011$ the authors $\quad 0270-6474 / 11 / 3111855-12 \$ 15.00 / 0$ throughout life (Burns et al., 2008; J. Burns and J. T. Corwin, unpublished observations), suggesting that the properties of the SC-SC junctions in mammalian ears may be responsible for restricting $\mathrm{HC}$ regeneration. Consistent with that notion, avian vestibular epithelia express little or no E-cadherin, but E-cadherin is strongly expressed in vestibular epithelia of rodents (Hackett et al., 2002; Kim et al., 2002, 2005; Warchol, 2007). Also, forced E-cadherin expression has been shown to inhibit the differentiation of certain HC-like characteristics in cell lines derived from the ear of the immortomouse (Hackett et al., 2002).

To determine whether and how the patterns of junctional cadherins are regulated, we investigated $\mathrm{N}$ - and E-cadherin in murine and human ears during postnatal maturation. Our results show that $\mathrm{N}$-cadherin is expressed in both the HC-SC and SC-SC junctions in vestibular epithelia and increases slightly with age, whereas E-cadherin is primarily restricted to SC-SC junctions and increases several-fold as mice mature. In addition, we discovered that $\gamma$-secretase inhibitor (GSI) treatments cause striolar SCs to internalize E-cadherin and then convert to a HC phenotype. GSI treatments are known to cause progenitor cells and SCs to become supernumerary HCs in embryonic and neonatal cochleae through inhibition of the Notch pathway (Yamamoto et al., 2006; Takebayashi et al., 2007; Hayashi et al., 2008; Doetzlhofer et al., 2009). In our experiments, GSI also appears to induce SC-to-HC conversion through Notch inhibition in the neonatal mouse utricles, but the robust SC-to-HC conversion we observed 
after striolar SCs internalized their E-cadherin suggests that a cell-autonomous linkage exists between the properties of SC junctions and the stability of the mammalian SC phenotype.

As mice mature, SC-SC junctions develop thicker F-actin belts and accumulate more E-cadherin. Between birth and P12, GSI treatments evoke progressively less E-cadherin internalization and less SC-to-HC phenotype conversion. Extrastriolar SCs have thicker F-actin belts and more junctional E-cadherin than SCs in the striola, and most do not deplete E-cadherin or convert after GSI treatments, but some do so after delays. The results provide support for the hypothesis that maturation of uniquely robust SC-SC junctions contributes to stabilization of the vestibular SC phenotype and limits HC replacement in mammalian ears.

\section{Materials and Methods}

Dissection of utricles. All animal experiments were performed according to protocols approved by the Animal Care and Use Committee at the University of Virginia. Swiss Webster mice of either sex were obtained from Charles River Labs and the Atoh1/nGFP transgenic line from Dr. Jane Johnson (University of Texas Southwestern Medical Center, Dallas, TX) (Lumpkin et al., 2003). Utricles were isolated from temporal bones in ice-cold DMEM/F-12 (Invitrogen) as described previously (Meyers and Corwin, 2007). Human utricles were collected during therapeutic labyrinthectomies on three adult patients. For their morphological analysis, we selected regions that showed the least hair cell depletion that likely resulted from surgical trauma.

Western blotting. We extracted protein from 10-15 pure utricular sensory epithelia, which were isolated by enzymatic digestion of the basement membrane and removal of the surrounding nonsensory epithelium as described previously (Montcouquiol and Corwin, 2001). For that, we centrifuged the tissue at 4000 relative centrifugal force $\times 10$ min at $4^{\circ} \mathrm{C}$, and the pellets were resuspended in a lysis buffer comprised of $10 \mathrm{~mm}$ Tris, pH 7.4, 400 mm NaCl, 1 mm EDTA, 1 mm EGTA, 1 mm NaF, 1\% Triton X-100, $10 \%$ glycerol, $0.1 \%$ SDS, $0.5 \%$ sodium deoxycholate, and protease inhibitor cocktail (P8340; Sigma-Aldrich). Western blots were performed as described previously (Lu and Corwin, 2008). Approximately $5 \mu \mathrm{g}$ of total protein per sample were loaded in duplicate $8 \%$ SDS-PAGE gels, separated by electrophoresis, and transferred to PVDF membranes (GE Healthcare). Membranes were immunoblotted with appropriate primary and secondary antibodies. Total actin was used as a loading control. Immunoreactive bands were visualized using enhanced chemiluminescence (ECL; GE Healthcare). Band intensity was measured and normalized against the intensity of the actin band measured from the same lane using ImageQuant TL 2005 and volume integration. The relative intensity of each of the bands was expressed as a percentage of the P1 sample level, with the value for the P1 band arbitrarily set to 100 . Immunoblotting experiments were replicated in four to five separate sample groups (10-15 utricles each).

Immunohistochemistry. Whole-mount utricles were fixed in $4 \%$ paraformaldehyde or Glyofixx (Thermo Fisher Scientific). To obtain 16-20 $\mu \mathrm{m}$ transverse cryostat sections, fixed tissue was processed through increasing concentrations of sucrose to $30 \%$ and then placed in Tissue Tek O.C.T. Compound and cooled to $-80^{\circ} \mathrm{C}$. To allow for comparison, utricles from the three ages examined (P1, P16, P82) were sectioned in one block of O.C.T. and processed together in the same solutions. Samples were blocked for $1 \mathrm{~h}$ at room temperature (RT) in PBS/0.02\% Triton X-100 (PBST) containing 10\% normal goat serum (Vector Laboratories) and then incubated overnight in the appropriate primary antibodies, followed by Alexa Fluor-conjugated secondary antibodies (Invitrogen). Samples were mounted in SlowFade (Invitrogen), and images were taken with a confocal microscope (Carl Zeiss LSM 510). For comparative studies across ages, the parameters of the scanning conditions for the laser scanning confocal microscope were set just below the image saturation level for the P82 samples, which always had the most intense labeling. Settings were held constant so that reliable comparisons could be made across replicates and age groups that were processed concurrently.

Primary antibodies and F-actin labeling. To label hair cells, we used rabbit or mouse anti-myosin VIIA (Proteus Biosciences or Developmen- tal Studies Hybridoma Bank) or rabbit anti-calretinin (Millipore Bioscience Research Reagents). We used mouse anti-bromodeoxyuridine (BrdU) (BD Biosciences) to label cells that had entered S-phase. We also used mouse anti-N-cadherin (clone 32; BD Biosciences), rabbit anti- $\beta$ catenin (Sigma-Aldrich, BD Biosciences), mouse anti-acetylated tubulin (Sigma-Aldrich), anti-spectrin (Millipore), rabbit anti-oncomodulin (Swant), anti-snail (Abcam), and anti-slug (Abgent). To label F-actin, utricles were incubated with Alexa Fluor-conjugated phalloidin $(5 \mathrm{U} / \mathrm{ml}$; Invitrogen) in PBST.

We used three different anti-E-cadherin antibodies, which all showed the same patterns under all experimental conditions: the mouse monoclonal anti-E-cadherin antibody from BD Biosciences (clone 36), produced using a human E-cadherin C-terminal (intracellular) recombinant protein, and the rat monoclonal anti-Uvomorulin/E-cadherin (clone DECMA-1; Sigma) and the rat anti-E-cadherin (clone ECCD-2; Zymed, Invitrogen), which both recognize the extracellular domain of E-cadherin.

RNA extraction and quantitative PCR. For RNA extraction, 8-10 utricles were pooled and dissolved in $500 \mu \mathrm{l}$ of Tri-Reagent RT (Molecular Research Center), and RNA was precipitated according to the protocol of the manufacturer using $2 \mu$ l of polyacryl carrier (Molecular Research Center). RNA was reverse transcribed using a High Capacity RNA-to-cDNA kit (Applied Biosystems). Quantitative PCR was performed in duplicates using a SensiMix SYBR Green and Fluorescein kit (Quantace) and gene-specific primer sets (Doetzlhofer et al., 2009) on a MyIQ/iCycler (Bio-Rad). Gene expression was analyzed using the Realtime PCR Miner algorithm (Zhao and Fernald, 2005). Cyclophilin gene (forward primer, CAGTGCTCAGAGCTCGAAAGT; reverse primer, GTGTTCTTCGACATCACGGC) was used as an endogenous reference and showed no significant change with treatments.

In vitro culture and treatments. Unroofed utricles were explanted nerve-side-down onto Cell Tak (2-4 $\mu$ l; BD Biosciences) on glass-bottom dishes (MatTek). The utricles were cultured in DMEM/F-12 containing 5\% fetal bovine serum (FBS) (Invitrogen), $0.25 \mu \mathrm{g} / \mathrm{ml}$ Fungizone (Invitrogen), and $10 \mu \mathrm{g} / \mathrm{ml}$ Ciprofloxacin (Bayer). To assess proliferation, $3 \mu \mathrm{g} / \mathrm{ml} \mathrm{BrdU}$ (Sigma-Aldrich) was added to the culture medium. Most cultured utricles were treated with $50 \mu \mathrm{M}$ of the $\gamma$-secretase inhibitor $N$ - $[N-(3,5-$ difluorophenacetyl-L-alanyl)]-S-phenylglycine $t$-butyl ester (DAPT) (Calbiochem, EMD) dissolved in DMSO, and control utricles were treated with $0.2 \%$ DMSO for the time indicated in Results. Similar results were obtained when utricles were treated with $5 \mu \mathrm{M}$ DAPT or the $\gamma$-secretase inhibitor X, L-685,458 \{1S-benzyl-4R-[1-(1S-carbamoyl-2-phenethylcarbamoyl)-1S-3methylbutylcarbamoyl]-2R-hydroxy-5-phenylpentyl $\}$ carbamic acid tert-butyl ester ( $5 \mu \mathrm{M}$; Calbiochem, EMD). Cycloheximide (40 $\mu \mathrm{M}$; SigmaAldrich) was used to block protein synthesis as shown in ears cultured from chickens (Zhao et al., 1996).

Scanning electron microscopy. DAPT- or vehicle-treated utricle cultures were fixed in $3 \%$ glutaraldehyde in $0.10 \mathrm{M}$ cacodylate buffer at $\mathrm{pH}$ 7.35 after 2,3 , and $5 \mathrm{~d}$ in vitro. After postfixation in $1 \% \mathrm{OsO}_{4}$ in the same buffer for $2 \mathrm{~h}$ at room temperature, the tissue was washed in cacodylate buffer and processed by the Osmium-Thiocarbohydrazide-Osmium method (Davies and Forge, 1987). Then, utricles were dehydrated through a graded ethanol series, critical point dried, mounted on stubs, and sputter coated with gold palladium. Samples were examined in a JEOL 6400 scanning electron microscope.

Measurement of apical junctional regions. Apical junctional region (AJR) width was measured as the perpendicular distance across the adherens junction and the circumferential F-actin belts in the two cells that shared each junction as described by Burns et al. (2008). We measured the widths of AJRs along lines that transected the extrastriolar and striolar regions as they ran from lateral-to-medial edges of the sensory epithelia. NIH ImageJ was used to trace the path of the line of hair bundle polarity reversal within the utricle and for recording the $x-y$ coordinates of each point on that line. Each AJR measurement was likewise associated with its $x-y$ coordinates, which were used to calculate the shortest distance to the line of polarity reversal. For each of four utricles, we measured $\sim 100$ AJRs in the region of sensory epithelium lateral to the line of reversal and $\sim 100$ AJRs in the region medial to it. The data were binned (bin width, $20 \mu \mathrm{m}$ ) and plotted as a function of the lateral-to-medial position in relation to the line of hair cell polarity reversal. 

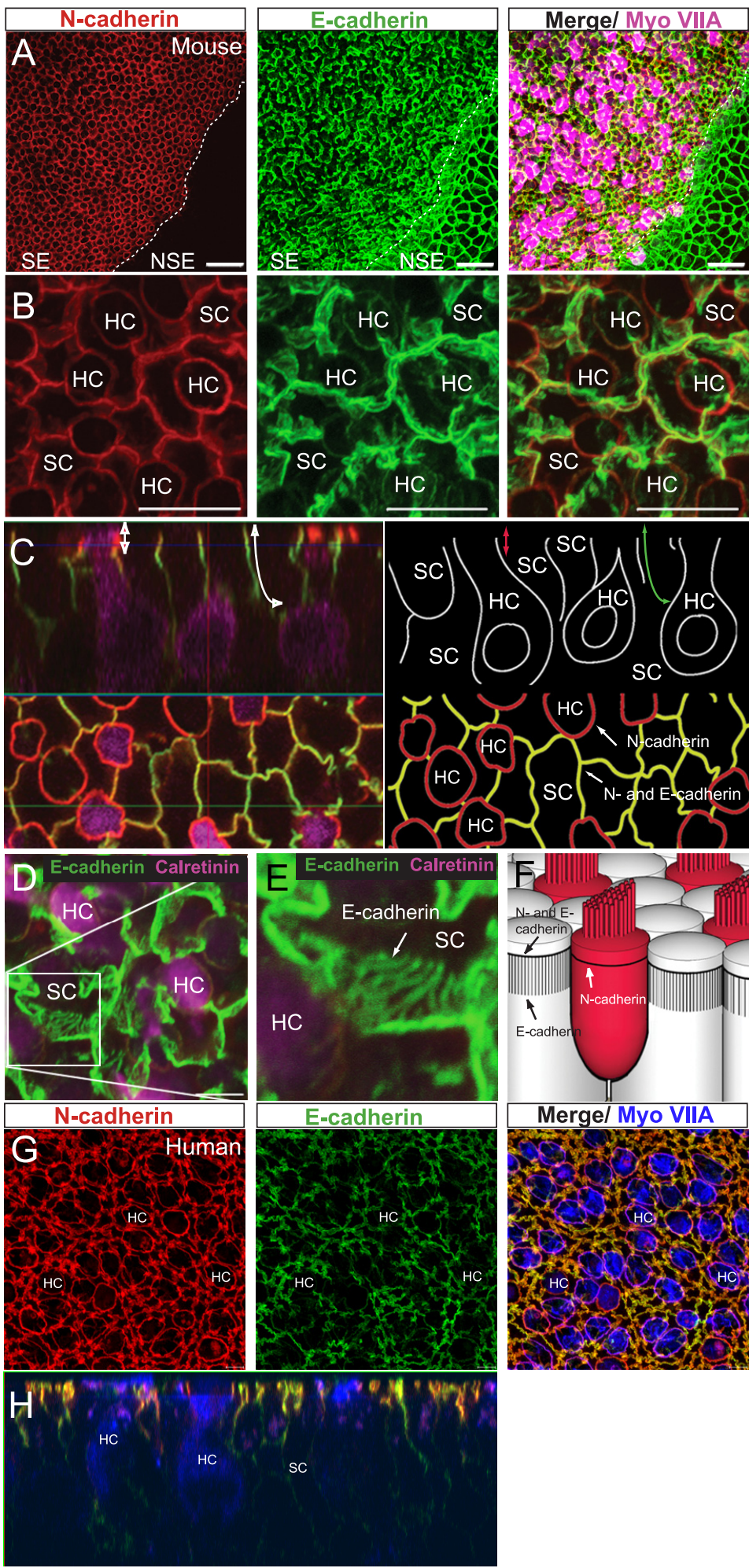

Figure 1. Cadherin localization in the mature mammalian utricle. $\boldsymbol{A}$, Immunolabeling of a whole-mount utricle from an adult mouse for N-cadherin (red), E-cadherin (green), and myosin VIIA (Myo VIIA, purple). Image shows part of sensory epithelium (SE, left) and nonsensory epithelium (NSE, bottom right). Dashed line delineates the border between SE and NSE. B, Maximal projection image of the apical surface of an adult utricular sensory epithelium immunostained for $\mathrm{N}$-cadherin (red) and E-cadherin (green). $\boldsymbol{C}$, Left, Section along the $z$-axis (top) and $x$ - $y$-axis (bottom) of an adult mouse utricle immunostained for $\mathrm{N}$-cadherin (red), E-cadherin (green), and calretinin (purple). Right, Schematic diagram of the image on the left. The long green arrow indicates the length of E-cadherin in the $z$-axis, and the short red arrow indicates the length of N-cadherin. $D, E$, Maximal projection image of adult mouse utricle immunostained for $\mathbf{E}$-cadherin and calretinin. $\boldsymbol{E}$ is an inset of $\boldsymbol{D}$ showing the detailed lines of $\mathbf{E}$-cadherin expression that descend along the basolateral membranes of SCS. F, 3D schematic of N-and E-cadherin localization in the utricular sensory epithelium. G, Maximal projection image showing the serpentine pattern of cadherins in an adult human utricular sensory epithelium. $\boldsymbol{H}$, Section along the $z$-axis of utricle in $\boldsymbol{G}$. Scale bars: $\boldsymbol{A}, 20 \mu \mathrm{m} ; \boldsymbol{B}, 10 \mu \mathrm{m} ; \boldsymbol{D}, 5 \mu \mathrm{m}$.
Quantification of area of conversion. Outlines were drawn around groups of converted cells using E-cadherin, N-cadherin, and myosin VIIA staining. The area inside each outlined region was calculated, and the areas of all the converted regions per utricle were then added and divided by the total area of the sensory epithelium (Ncadherin, myosin VIIA positive). Results were expressed as the percentage of the sensory epithelium where conversion occurred.

Data analysis. Each experiment was repeated at least three times and analyzed by $t$ test or one-way ANOVA (GraphPad Prism; GraphPad Software). The figures illustrate images that we carefully selected to show the average effect obtained for each experimental condition.

\section{Results}

$\mathrm{N}$-cadherin is in all the junctions, but SC-SC junctions in mouse and human utricles also contain E-cadherin

In utricles from adult mice, we found strong immunostaining for $\mathrm{N}$-cadherin at the HC-SC and SC-SC junctions within the sensory epithelium but not in the nonsensory epithelium (Fig. 1A,B). E-cadherin, in contrast, is expressed strongly in both the sensory epithelium and the surrounding nonsensory epithelium. Within individual SCs, E-cadherin immunostaining is much stronger at the $\mathrm{SC}-\mathrm{SC}$ junctions than at the HC-SC junctions (Fig. $1 B$ ) (Hackett et al., 2002; Kim et al., 2002, 2005). Also in adults, N-cadherin appears to be limited to the zonula adherens, whereas E-cadherin extends from the zonula adherens basalward in complex patterns (Fig. 1C,D).

Immunostaining of adult human vestibular organs showed high levels of $\mathrm{N}$-cadherin at HC-SC and SC-SC junctions, as in mice, and low levels in the band of nonsensory epithelium immediately adjacent to the sensory macula but none in the roof epithelium (Fig. 1G) (data not shown). SC-SC junctions in human utricles labeled strongly for E-cadherin, with the labeling extending beneath the classic zonula adherens, as in mice, but differed in that they followed distinctly serpentine courses (Fig. $1 G, H)$. The cell junctions in the human nonsensory and roof epithelia also labeled for E-cadherin.

\section{E-cadherin levels increase as SC-SC} junctions mature postnatally

To assess changes in expression and subcellular distribution, we immunolabeled whole-mount and cryosectioned utricles from 1-d-old (P1), 16-d-old (P16), and adult (P82) mice with antibodies to $\mathrm{N}$-cadherin and E-cadherin, the linker $\beta$-catenin, and/or the $\mathrm{HC}$ marker myosin VIIA. With postnatal maturation, junc- 

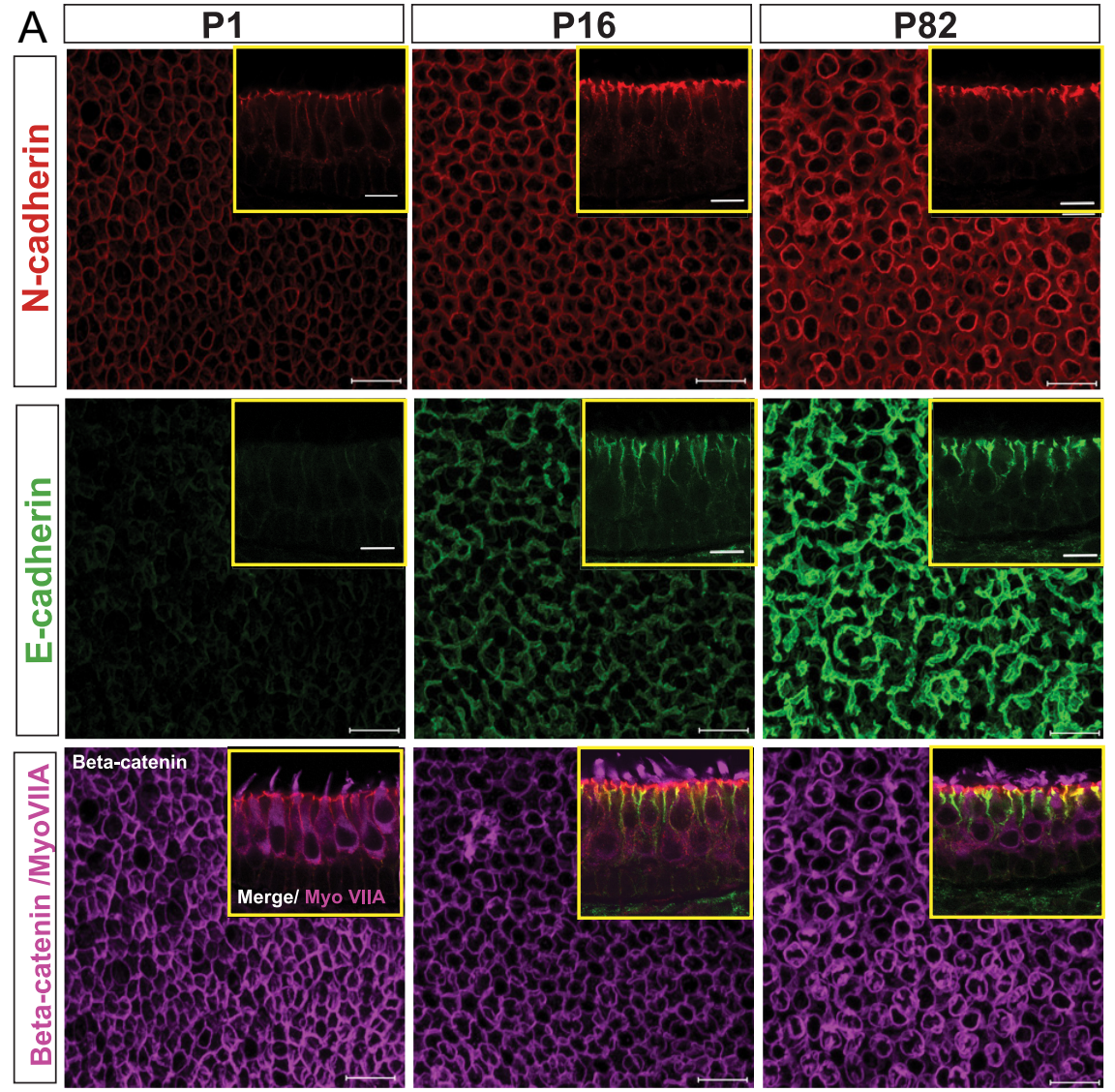

B
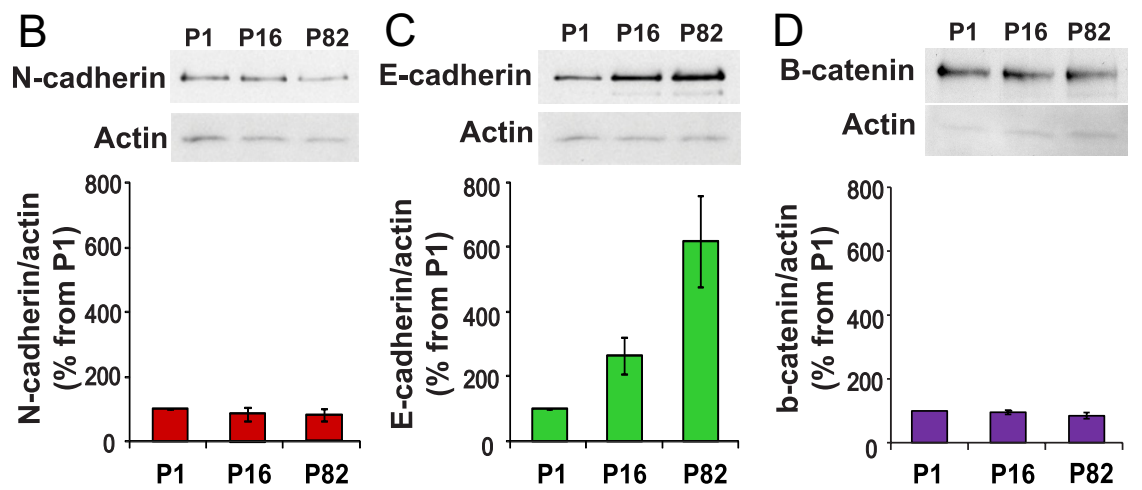

\section{E}

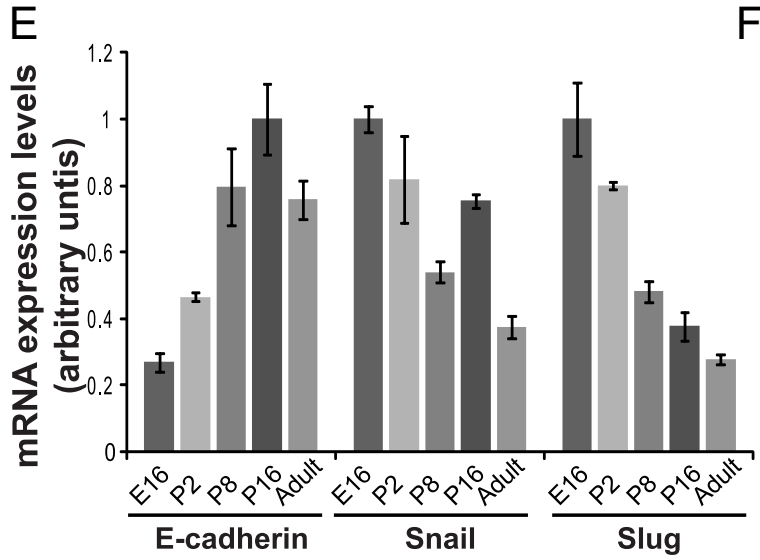

$\mathrm{F}$
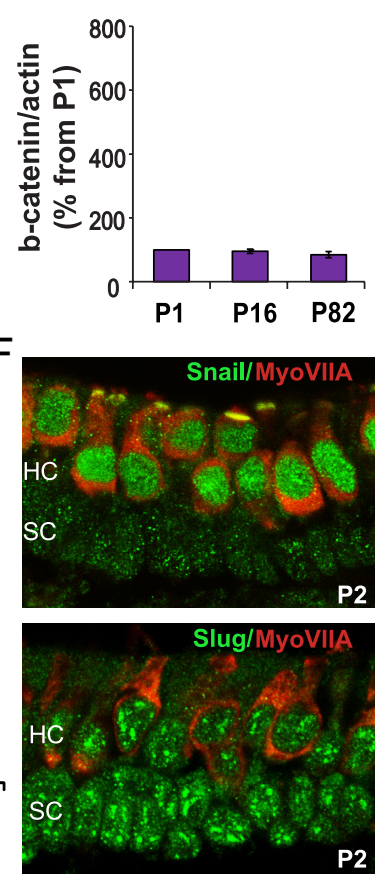

Figure 2. Levels of E-cadherin at the SC-SC junctions increase as mice mature postnatally. $\boldsymbol{A}$, Representative images of sensory epithelium in whole-mount utricles from P1, P16, and P82 mice immunolabeled for N-cadherin (red), E-cadherin (green), and $\beta$-catenin (purple). Insets, Representative images of cryostat sections of utricles from P1, P16, and P82 mice immunolabeled for $\mathrm{N}$-cadherin (red), E-cadherin (green), and myosin VIII (purple). Scale bars, $10 \mu \mathrm{m} . \boldsymbol{B}-\boldsymbol{D}$, A representative example of a Western blot showing levels of $\mathrm{N}$-cadherin $(\boldsymbol{B})$, $\mathrm{E}$-cadherin $(\boldsymbol{C}), \boldsymbol{\beta}$-catenin $(\boldsymbol{D})$, and total actin (internal control) in pure delaminated utricular tional $\mathrm{N}$-cadherin expression increased modestly, and its subcellular distribution became restricted. In P1 utricles, low $\mathrm{N}$-cadherin labeling extended throughout the basolateral membranes of the HCs and SCs, but it became more concentrated at the level of the zonula adherens at P16 and P82 (Fig. 2A, insets).

In contrast, E-cadherin expression increased greatly at the SC-SC junctions during the weeks after birth and on into adulthood (Fig. 2A). Under fixed scanning conditions, E-cadherin immunofluorescence was barely detectable in P1 sensory epithelia but was so intense at P82 that the confocal images approached saturation. The increase in E-cadherin intensity observed between P16 and P82 appeared to result primarily from greater concentration at the level of the zonula adherens, not from changes below that depth (Fig. $2 A$, insets). $\beta$-Catenin was localized to all the HC-SC and SC-SC junctions, but $\beta$-catenin immunostaining did not change appreciably between young and adult utricles (Fig. 2A).

Relative immunoblot band intensities for total $\mathrm{N}$-cadherin protein in utricular sensory epithelia did not show a significant change between $\mathrm{P} 1$ and $\mathrm{P} 16$ or P1 and P82 (Fig. $2 B$ ). However, total E-cadherin in the same samples increased by $>250 \%$ between $\mathrm{P} 1$ and $\mathrm{P} 16$ and by $>600 \%$ from P1 to P82 $(263 \pm 57 \%, n=5, p=0.04$; and $619 \pm$ $141 \%, n=5, p=0.02$, respectively) (Fig. $2 C)$. Thus, the adhesive (E-cadherin) and cytoskeletal (F-actin) components of the SC-SC junctions in mammalian balance epithelia both increase from birth to adulthood. Repeated, separate immunoblot experiments confirmed that the levels of $\beta$-catenin remained unchanged across the P1, P16, and P82 age range (Fig. 2D).

E-cadherin mRNA increases during the first postnatal week

In utricles harvested from E16, P2, P16, and adult (P69-P73) mice, we found that the levels of E-cadherin mRNA increased with age. Transcript levels in older ani-

\section{$\leftarrow$}

sensory epithelia harvested from P1, P16, and P82 mice. Bottom, Summary of quantification of experiments. Levels of $\mathrm{N}$-cadherin, E-cadherin, and $\beta$-catenin were normalized to total actin levels, and values were expressed as a percentage relative to $\mathrm{P} 1$ for comparison. Average percentages relative to P1 are shown $(n=5)$. $\boldsymbol{E}$, Relative mRNA expression levels of E-cadherin, Snail, and Slug in E16, P2, P8, P16, and adult utricles ( $n=3$ for each age). mRNA levels for each gene were made relative to the highest for comparison. $F$, Images of cryostat sections of utricles from P2 mice immunolabeled for myosin VIIA (red), snail (green, top), or slug (green, bottom). 
mals (P8, P16, and adult) were significantly greater than in embryonic and neonatal mice (E16 and P2; one-way ANOVA, $p<$ 0.05 ) (Fig. 2E), so we measured mRNA levels for Snail and Slug, two potent repressors of E-cadherin transcription. At P8 and in adults, Snail mRNA levels were significantly lower than at E16, P2, and P16 (one-way ANOVA, $p<0.05$ ) (Fig. $2 E$ ), but those levels did not correlate with mRNA levels for E-cadherin ( $p=$ 0.26 , Pearson's $r=-0.62$ ). However, the levels of Slug mRNA decreased progressively with age. Levels at P8, P16, and in adulthood were significantly lower than at E16 and P2 (one-way ANOVA, $p<0.05$ ) (Fig. $2 E$ ) and correlated inversely with $E$-cadherin mRNA levels across the ages investigated $(p<0.05$, Pearson's $r=-0.91)$. That correlation was even stronger from E16 to P16 (Pearson's $r=-0.99$ ), suggesting that the postnatal E-cadherin increase in SCs stems from decreased expression of the transcriptional repressor Slug. Consistent with this hypothesis, immunostaining in embryonic and neonatal mouse utricles showed high levels of Slug in HC and SC nuclei, in which it could repress E-cadherin expression (Fig. 2 F). In contrast, Snail expression was very low in SCs and high in HCs (Fig. $2 F$ ). With continuing postnatal maturation, the immunostaining for Slug and Snail both decreased, as did mRNA levels (data not shown).

\section{Inhibitors of $\gamma$-secretase induce cell phenotype changes in the striola}

When cochleae of embryonic and neonatal mice have been treated with GSIs, progenitor cells or immature SCs have been induced to convert directly to a HC phenotype, without intervening cell divisions (Yamamoto et al., 2006; Takebayashi et al., 2007; Hayashi et al., 2008; Doetzlhofer et al., 2009). Because our findings show that E-cadherin is specifically expressed in SCs in mammalian balance epithelia (Fig. 1) and E-cadherin has been reported to inhibit some HCs characteristics when overexpressed in cell lines (Hackett et al., 2002), we hypothesized that E-cadherin expression in SCs might limit their capacity to change phenotype and convert into HCs. We addressed this hypothesis by treating P2 mouse utricles with the GSI DAPT (50 $\mu \mathrm{M})$ or vehicle for $30 \mathrm{~h}$ and then continuing to culture them in control medium. Striola regions of the DAPT-treated utricles cultured for a total of $72 \mathrm{~h}$ contained appreciable numbers of cells that were characterized by circular apical surface outlines and villous projections that were noticeably longer than microvilli typical of SCs (Fig. 3B). Such cells were even more prominent in $120 \mathrm{~h}$ cultures (Fig. $4 B$ ). Consistent with early stages of cuticular plate formation and conversion to HC phenotypes, these cells exhibited light but distinctly positive immunostaining for the $\mathrm{HC}$ markers myosin VI and myosin VIIA and the cuticular plate marker spectrin (Fig. 3B). Also, they no longer exhibited the cytokeratin immunostaining found in the striolar SCs of controls (data not shown). A single acetylated-tubulin-positive kinocilium, which was distinctly longer than the primary cilium typical of SC surfaces, projected from each of those circular cell surfaces (data not shown).

Scanning electron microscopy showed that the striola of utricles treated for $30 \mathrm{~h}$ with DAPT and cultured for $48 \mathrm{~h}$ in total $(n=13)$ contained numerous small circular cell surfaces that were filled by dense accumulations of thickened and elongate microvilli. Many of these cells were in direct contact with other cells that had the similar small hair-bundle-like surface characteristics (Fig. 4D, arrowheads). A single cilium was at the center of the surface in some of these cells and closer to one side in others (Fig. $4 D)$. In utricles cultured for $72 \mathrm{~h}(n=8)$, the microvilli on such cells were noticeably longer. Those on comparable cells in
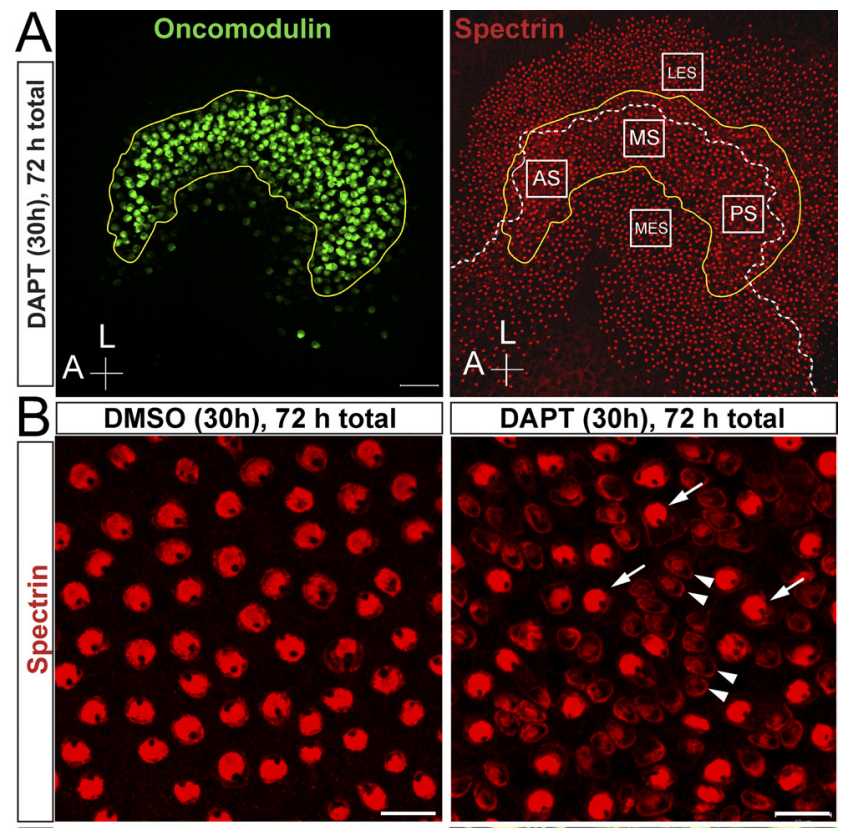

DAPT (30h), $72 \mathrm{~h}$ total
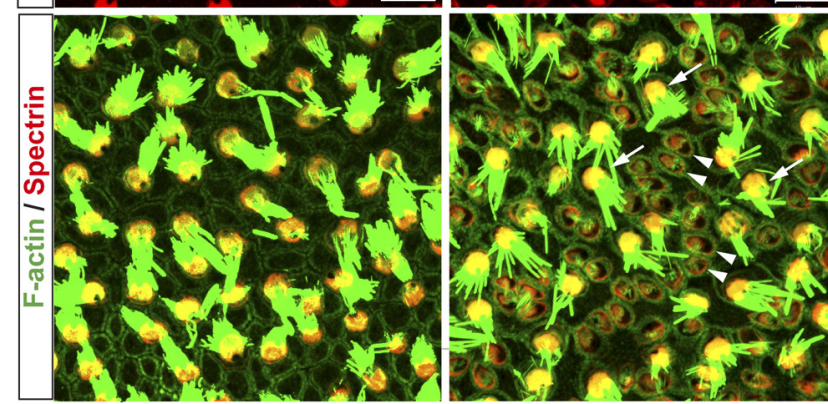

C

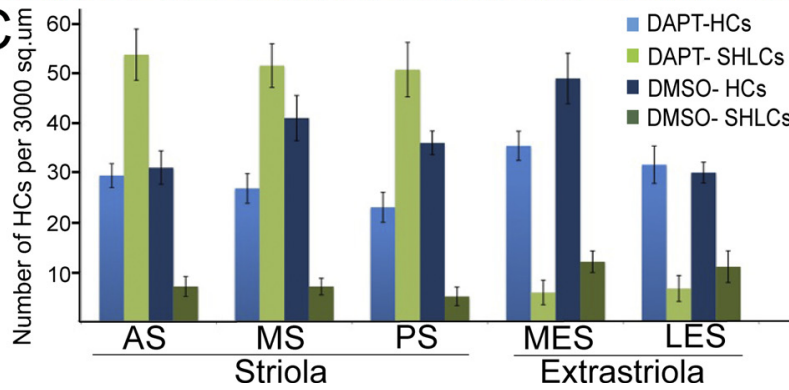

Figure 3. The $\gamma$-secretase inhibitor DAPT induces a rapid and robust phenotypic conversion of striolar supporting cells into hair cells in neonatal utricles. $\boldsymbol{A}$, Maximal projection image of a DAPT-treated utricle labeled for oncomodulin (green) and spectrin (red). The striolar region (yellow line) was delineated using oncomodulin as a marker. The line of polarity reversal (white dashed line) was drawn using spectrin labeling of cuticular plates. The image also illustrates the size of the $3000 \mu \mathrm{m}^{2}$ areas that were quantified by counting preexisting HCs and SHLCs (anterior striola, AS; middle striola, MS; posterior striola, PS; medial extrastriola, MES; lateral extrastriola, LES). $\boldsymbol{B}$, Higher-magnification image of a DMSO-treated and a DAPT-treated utricle labeled for spectrin (red) and F-actin (green) at the level of the striola. Arrowheads point to the newly generated hair-cell-like cells and arrows point to mature, preexisting hair cells. Scale bars: $A, 50 \mu \mathrm{m} ; \boldsymbol{B}, 10 \mu \mathrm{m}$. C, Quantification of the number of preexisting HCs and SHLCs per $3000 \mu \mathrm{m}^{2}$ in utricles treated with DAPT or vehicle for the first $30 \mathrm{~h}$ and cultured for a total of $72 \mathrm{~h}$.

GSI- treated utricles cultured for $120 \mathrm{~h}(n=8)$ had the beveled, "staircase" appearance of sensory hair bundles (Fig. 4D). Such small HC-like cells (SHLCs) resembled embryonic HCs at early stages of differentiation (Mbiene et al., 1984; Favre et al., 1986; Forge et al., 1997; Denman-Johnson and Forge, 1999). All of these bundles were distinctly shorter than the large, more common, presumably preexisting hair bundles in those utricles and in the DMSO controls. 
Colabeling with anti-spectrin and antioncomodulin, which labels type I vestibular HCs (Simmons et al., 2010), confirmed that most of the SHLCs in the DAPT-treated utricles were in the striola (Fig. $3 A$, yellow line), a crescent-shaped region of the macula in which hair bundle polarity changes by $180^{\circ}$ (Fig. 3A, white dashed line) (Li et al., 2008). We identified SHLCs (arrowheads) by their low immunoreactivity for myosin VIIA and spectrin, proteins that are abundant in preexisting HCs (arrows) and completely absent from the surrounding SCs. We counted SHLCs in five areas in the anterior, medial, and posterior striola, and in medial and lateral extrastriolar regions of each macula (Fig. 3A). Counts showed that the striola regions of DAPT-treated utricles averaged $51 \pm 3$ SHLCs per $3000 \mu \mathrm{m}^{2}(30 \mathrm{~h}$ DAPT, fixed after $72 \mathrm{~h}$ total, $n=5$ ), whereas vehicle-treated controls averaged $4 \pm 1 \mathrm{SH}$ LCs per $3000 \mu \mathrm{m}^{2}(n=6)$ (Fig. 3C). The extrastriolar regions in the DAPT-treated and the vehicle control utricles both average $6 \pm 2$ SHLCs per $3000 \mu \mathrm{m}^{2}$. Thus, SHLC density in the striola of the GSI-treated utricles is 8.5-12 times that observed in controls and more than eight times the density in the extrastriola regions in both treated and control utricles.

\section{Small HC-like cells increase at the expense of SC numbers}

To determine whether the SHLCs arose through cell division, we cultured DAPTtreated and control utricles with BrdU. Immunostaining showed that scattered SCs contained BrdUpositive nuclei, but no SHLCs were BrdU positive (Fig. 4C). Therefore, we assessed the possibility that striolar SHLCs arose from SCs that converted to the $\mathrm{HC}$ phenotype without passing through S-phase of an intervening cell cycle. For this, we counted the SCs, preexisting HCs, and SHLCs in the striola regions of DAPT-treated utricles and vehicle controls. The DAPT-treated utricles contained many SHLCs (arrowheads) in the spaces between the large, preexisting HCs in the striola (arrows) $\left(60 \pm 13\right.$ and $17 \pm 2$ per $3000 \mu \mathrm{m}^{2}$, respectively) (Fig. $4 B$ ). Such DAPT-treated utricles contained more than two times the summed density of striolar HCs and HC-like cells that were present in the vehicle controls $(77 \pm 13$ vs $35 \pm 3 \mathrm{HCs}$ and SHLCs, $p<0.05 ; n=4$ utricles per group). As those HC densities increased, the density of SCs in the DAPT-treated utricles decreased to $43 \%$ of the SC density we measured in matched regions of the vehicle controls ( $35 \pm 5$ vs $82 \pm 1$ SCs per $3000 \mu \mathrm{m}^{2}$, respectively, $p=0.002 ; n=4$ utricles each) (Fig. $4 A, B$ ). The total counts of cells per $3000 \mu \mathrm{m}^{2}$ did not differ significantly between the treated and control samples, consistent with the likelihood that the SHLCs in the striola of the DAPT-treated utricles increased at the expense of SC numbers, as would be expected if SCs were converting directly to a HC phenotype.

DAPT treatments lead to downregulation of Hes and Hey transcripts and induce Atoh1 expression in striolar SCs In developing ears, the inhibition of $\gamma$-secretase is believed to block cleavage of ligated Notch, which prevents release of the
Notch intracellular domain (NICD) and thereby influences cell fate (Yamamoto et al., 2006; Takebayashi et al., 2007; Hayashi et al., 2008; Doetzlhofer et al., 2009). In the nucleus, NICD binds the RBPJ (CBF1) repressor complex, which leads to upregulated expression of Hes and Hey. Hes and Hey bHLH proteins, in turn, suppress transcription of the prosensory bHLH transcription factor Atoh1. In embryonic ears, Atoh1 expression is necessary and sufficient for HC fate determination (Bermingham et al., 1999; Zheng and Gao, 2000; Woods et al., 2004; Gubbels et al., 2008).

To investigate whether GSI treatments inhibit the Notch pathway as they induce utricular SCs to convert into HCs, we compared mRNA levels for Hes1, Hes5, Hey1, Hey2, HeyL, and Atoh1 in utricles cultured for 18 and $30 \mathrm{~h}$ with DAPT or DMSO. In the DAPT-treated utricles, mRNA levels for all of those Hes and Hey genes decreased progressively and significantly compared with matched vehicle-control samples (Fig. $5 A$ ) ( $p<0.05$; three replicate experiments for all genes and time points). In addition, levels of Atoh1 mRNA in the DAPT-treated utricles were 3.7 and 4.7 times the levels in matched controls at the 18 and $30 \mathrm{~h}$, respectively (Fig. 5A) ( $p<0.05, n=3$ for each). These results are consistent with the hypothesis that DAPT acts to induce SCto-HC conversion in postnatal utricles, in part, by inhibiting Notch signaling.

To identify and localize cells that were upregulating Atoh1, we cultured utricles from neonatal Atoh1/nGFP mice, which express nuclear EGFP under control of the Atoh1 promoter (Lumpkin et al., 2003), in the constant presence of $50 \mu \mathrm{M}$ DAPT or vehicle 

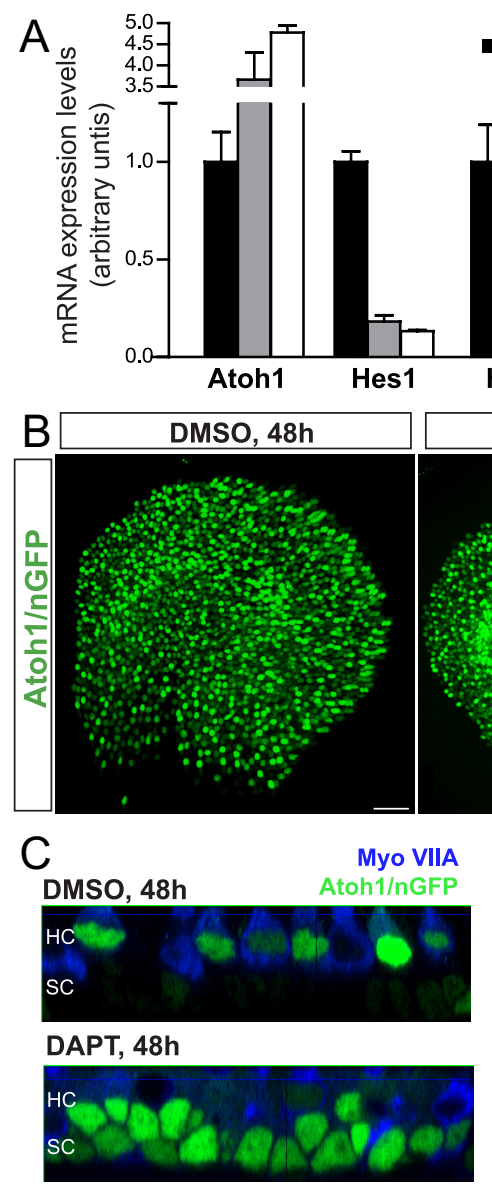

- Control aDAPT 18h aDAPT 30h

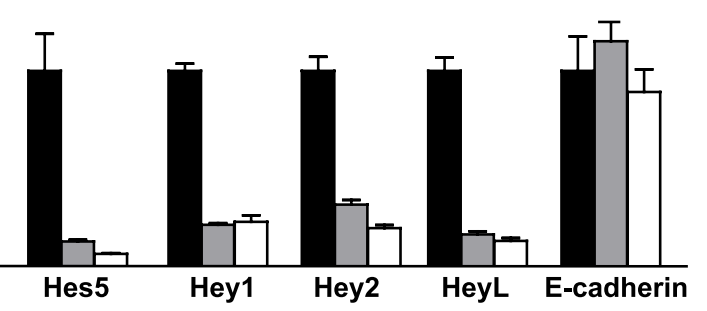

DAPT, 24h
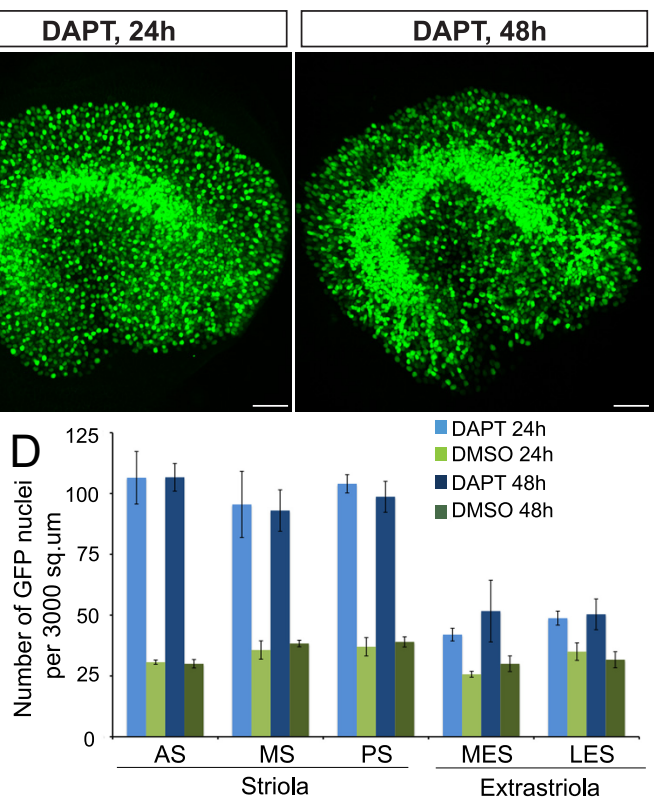

Figure 5. DAPT induces downregulation of Hes and Hey genes and Atoh 1 expression in striolar SCS. A, Relative mRNA expression levels for Atoh1, Hes1, Hes5, Hey1, Hey2, HeyL, and E-cadherin in P2 utricles treated with DAPT or vehicle (DMSO) for 18 and $30 \mathrm{~h}$ ( $n=3$ for each gene). mRNA levels for each gene were plotted relative to their respective vehicle control mRNA levels. $\boldsymbol{B}$, Atoh 1 expression in whole-mount P2 Atoh $1 / \mathrm{nGFP}$ utricles treated with vehicle for $48 \mathrm{~h}$ (left) or DAPT for $24 \mathrm{~h}$ (middle) or $48 \mathrm{~h}$ (right). Scale bars, $50 \mu \mathrm{m}$. C, Images showing $z$-axis of Atoh $1 / n$ GFP fluorescence in P2 utricles treated with DMSO or DAPT and immunostained for myosin VIIA (blue). Images taken at the level of striola. D, Quantification of GFP-positive cells in five $3000 \mu \mathrm{m}^{2}$ regions (anterior striola, AS; middle striola, MS; posterior striola, PS; medial extrastriola, MES; lateral extrastriola, LES) of P2 utricles treated with DAPT or DMSO for two durations.

until fixation at 15,24 , or $48 \mathrm{~h}$. GFP-positive nuclei that were distinctly smaller than the more apical HC nuclei were first detected in the basal SC nuclear layer of the striola at $15 \mathrm{~h}(n=4)$ and became more evident after $24 \mathrm{~h}$ of DAPT treatment (Fig. $5 B, C)$. By $48 \mathrm{~h}$, Atoh1-GFP expression expanded to the edges of the striola and perhaps into the peristriolar regions (Fig. $5 B, C$ ), but most of the extrastriolar regions showed only scattered SCs expressing nGFP. It appears that, in utricles as young as P2, most extrastriolar SCs are not responsive to the GSI treatments that induce substantial amounts of conversion in striolar SCs (Fig. 5B,C). Both the control and treated Atoh1/nGFP utricles contained scattered large GFP-positive nuclei within myosin-VIIA-positive cells that appear to be newly differentiating $\mathrm{HCs}$, but control Atoh1/nGFP utricles did not contain GFP-positive SC nuclei $(n=4)$ (Fig. $5 C$ ). We assessed Atoh1 expression by immunostaining as well, which showed that DAPT treatment induced Atoh1 expression mainly in the striolar SCs, validating the results obtained in utricles from the Atoh1/nGFP mice (data not shown).

In $\mathrm{P} 2$ utricles treated with DAPT for $24 \mathrm{~h}$, the striola averaged $102 \pm 6$ Atoh1-GFP-positive cells nuclei per $3000 \mu \mathrm{m}^{2}$ in regions that sampled the anterior, middle, and posterior striola, whereas medial and lateral extrastriolar regions averaged $45 \pm 2$ per 3000 $\mu \mathrm{m}^{2}$ (Fig. 5D). In contrast, vehicle control utricles averaged $34 \pm$
2 GFP-positive nuclei per $3000 \mu \mathrm{m}^{2}$ in the striola after $24 \mathrm{~h}$ and $30 \pm 3$ per $3000 \mu \mathrm{m}^{2}$ in their extrastriolar regions. Counts within the $3000 \mu \mathrm{m}^{2}$ sampled areas remained similar after culturing with DAPT or vehicle for $48 \mathrm{~h}$ (Fig. 5D), indicating that the incidence of responding cells within the sampled regions saturates by $24 \mathrm{~h}$. As with the preceding evidence, these results are consistent with the idea that DAPT treatments block Notch signaling, thereby alleviating ongoing repression of Atoh1 transcription that appears to be required for active maintenance of the SC phenotype in the striola of young mice.

\section{Striolar SCs internalize E-cadherin and express myosin VIIA, without detectable depletion of $\mathrm{N}$-cadherin}

We used immunostaining to investigate what happens to junctional cadherins when epithelial cells change from a SC phenotype to a $\mathrm{HC}$ phenotype. In utricles cultured with DAPT for $18 \mathrm{~h}$ or more, many striolar SCs exhibited considerably less junctional E-cadherin than the extrastriolar SCs in the same epithelia (Fig. $6 A, B)$. At $24 \mathrm{~h}$, the apical cytoplasm of many striolar cells contained puncta that were intensely positive for E-cadherin (arrows), but such cells maintained control levels of junctional N-cadherin (Fig. 6C,D). Punctate cytoplasmic immunostaining patterns were obtained with antibodies that separately bound only to the intracellular and only to the extracellular domains of E-cadherin, indicating that both domains are internalized. It appears that SCs that are responding to inhibition of $\gamma$-secretase selectively internalize E-cadherin from their adherens junctions by a mechanism that allows $\mathrm{N}$-cadherin to remain in the junctional membrane. In addition, quantitative RT-PCR showed no change in E-cadherin mRNA levels between DAPTtreated utricles and vehicle controls (Fig. 5A).

After $48 \mathrm{~h}$ of continuous DAPT treatment, most of the striolar SCs that had reduced junctional E-cadherin also expressed the $\mathrm{HC}$ marker myosin VIIA, but such cells still retained the elongate shape of SCs, extending from the apical surface to the basal lamina (Fig. $6 C, D)$. Most striolar SCs in utricles from Atoh $1 / \mathrm{nGFP}$ reporter mice also exhibited reduced junctional E-cadherin, became GFP positive, and immunostained for myosin VIIA after 48 of DAPT (Fig. 6E). However, a few SCs in the striola regions and most SCs in the extrastriolar regions of these utricles did not downregulate E-cadherin by $48 \mathrm{~h}$. In all cases, those cells failed to express Atoh1-GFP or myosin VIIA (Fig. 6C-E). Thus, Atoh1 induction and phenotypic conversion into HCs appear to be tightly correlated with E-cadherin internalization in SCs.

\section{The GSI-induced internalization of E-cadherin requires protein synthesis}

To test whether inhibition of $\gamma$-secretase induced E-cadherin internalization via signaling that depended on translation, we treated utricles with DAPT and cycloheximide $(40 \mu \mathrm{M})$ for $30 \mathrm{~h}$, followed by 
$42 \mathrm{~h}$ in control medium. In contrast to utricles treated with DAPT alone, in which striolar SCs exhibited pervasive downregulation of E-cadherin, as well as expression of myosin VIIA and other signs of SC-to-HC conversion (Fig. $7 A)(n=3)$, the striolar SCs in utricles treated with cycloheximide and DAPT together failed to internalize junctional E-cadherin and failed to convert to a HC phenotype $(n=4)$. Controls treated with cycloheximide alone showed no detectable effects on the integrity or organization of the sensory epithelium $(n=3)$. The dependence on protein synthesis raised the question of whether E-cadherin internalization is downstream of Atoh1 expression. When we examined utricles treated with DAPT for only $15 \mathrm{~h}$, however, we did not find any Atoh1/nGFP-positive cells that retained strong labeling for junctional E-cadherin (data not shown). Such a finding would have indicated that Atoh1 expression precedes the changes in E-cadherin at the cell junctions.

\section{Longer GSI treatments induce more SC-to-HC conversion}

When we extended the DAPT treatment of P2 utricles to the full $72 \mathrm{~h}$ culture period, almost all the SCs in the striola converted into HCs (Fig. 7B). The SCs in control utricles and those in the regions of the utricle that are unresponsive to the DAPT treatments had elongated hourglass-shaped somata that extended down to the basal lamina and anchored the sensory epithelium to it. In contrast, many of the phenotypically converting SCs exhibited the goblet-shaped somata that are characteristic of HCs. The somata of those cells terminated in rounded basal ends that were well above the basal lamina. The pervasiveness of the SC-to-HC conversion that occurred with the longer DAPT treatments caused large patches of the still living striolar epithelium to blister up, eventually creating holes in the sensory epithelium (Fig. 7B, arrows). After 72-h and longer DAPT treatments, some extrastriolar SCs internalized E-cadherin and began converting into HCs, but many did not, which suggests that SCs differ regionally and individually in their capacity for changing phenotype.

\section{Striolar SCs have thinner}

\section{circumferential F-actin belts than}

\section{extrastriolar SCs}

Because differences in the incidence of SC-to-HC phenotype conversion between the striola and extrastriola regions were strikingly consistent, we looked for structural correlates and found that E-cadherin immunostaining in the extrastriolar regions of neonatal control utricles was more intense than in the

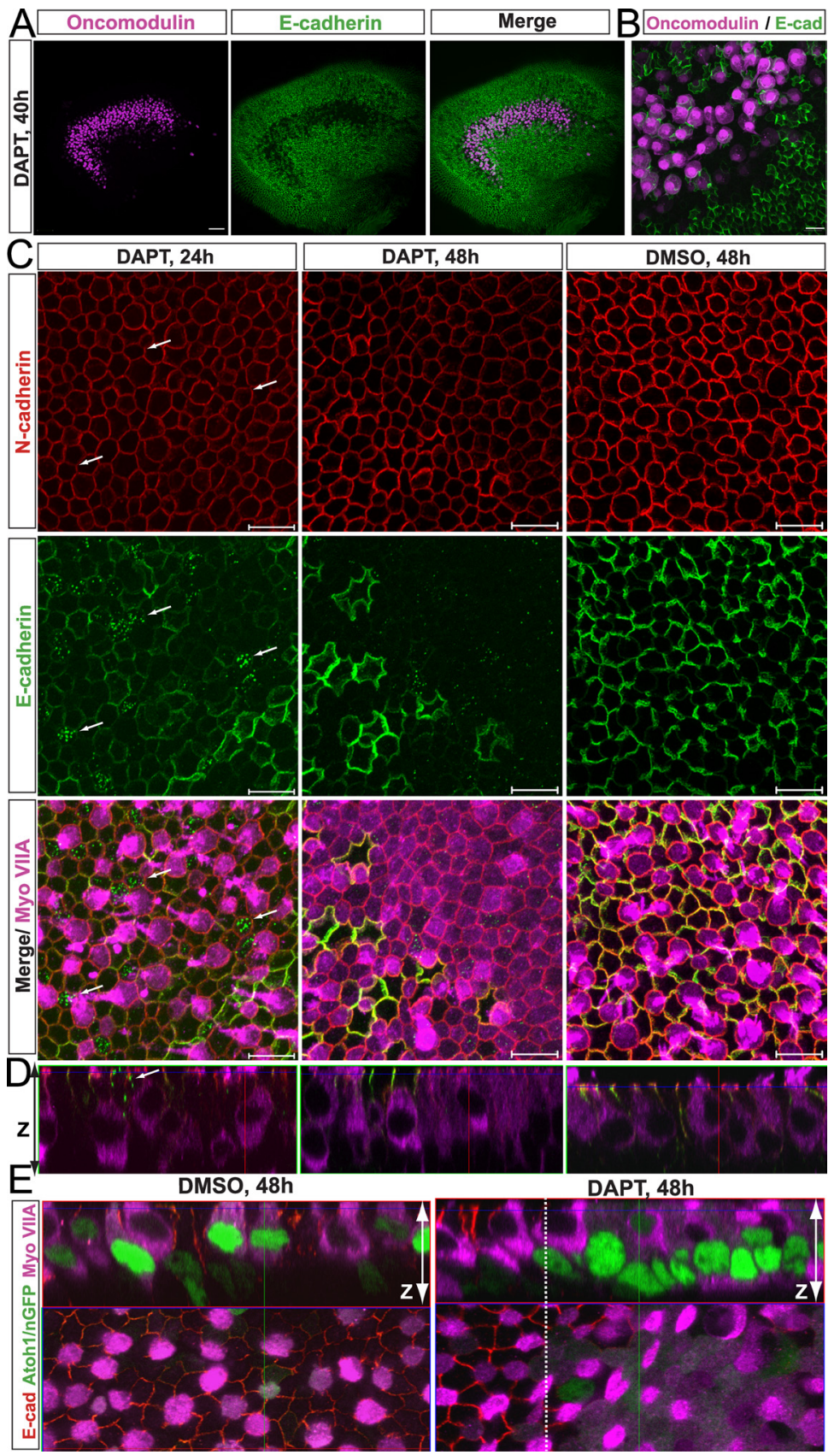

Figure 6. DAPT induces downregulation of E-cadherin and subsequent upregulation of myosin VIIIA in striolar SCs. A, Image of a utricle treated with DAPT for $40 \mathrm{~h}$ and immunostained for oncomodulin (purple) and E-cadherin (green). Depletion of E-cadherin occurs specifically in the striolar region, which shows immunostaining for the type I hair cell marker oncomodulin. Scale bar, 50 $\mu \mathrm{m} . \boldsymbol{B}$, Higher-magnification image of the striolar region. Scale bar, $10 \mu \mathrm{m}$. C, Higher-magnification images of the striolar sensory epithelium. Arrows point to cells that show E-cadherin internalization. Myosin VIIA is upregulated after $48 \mathrm{~h}$ of DAPT treatment in cells of the striola. Scale bars, $10 \mu \mathrm{m}$. D, Images showing z-axis of sensory epithelium of utricles treated with vehicle or DAPT for 24 or $48 \mathrm{~h}$. Arrow points to cells that show E-cadherin internalization. $E$, Images showing $x-y$-axis and $z$-axis of Atoh $1 / \mathrm{nGFP}$ P2 utricles treated with vehicle (DMSO) or DAPT and immunostained for E-cadherin (red) and myosin VIIA (purple). The image of the DMSO control utricle was taken at the striolar/peristriolar boundary. In the image of the DAPT-treated utricle, the extrastriolar region is to the left of the white dashed line, and the striolar region is to the right.

striola (Fig. 8A). Then we labeled F-actin in P2 mouse utricles with fluorescent phalloidin and measured the widths of the AJRs. AJRs comprise an apical intercellular junction and the circumferential F-actin belts that bracket each side (Burns et al., 2008). When we plotted AJR widths as a function of the lateral-tomedial distance from the line of hair cell polarity reversal, the results revealed that AJRs of SC-SC junctions in the extrastriolar 

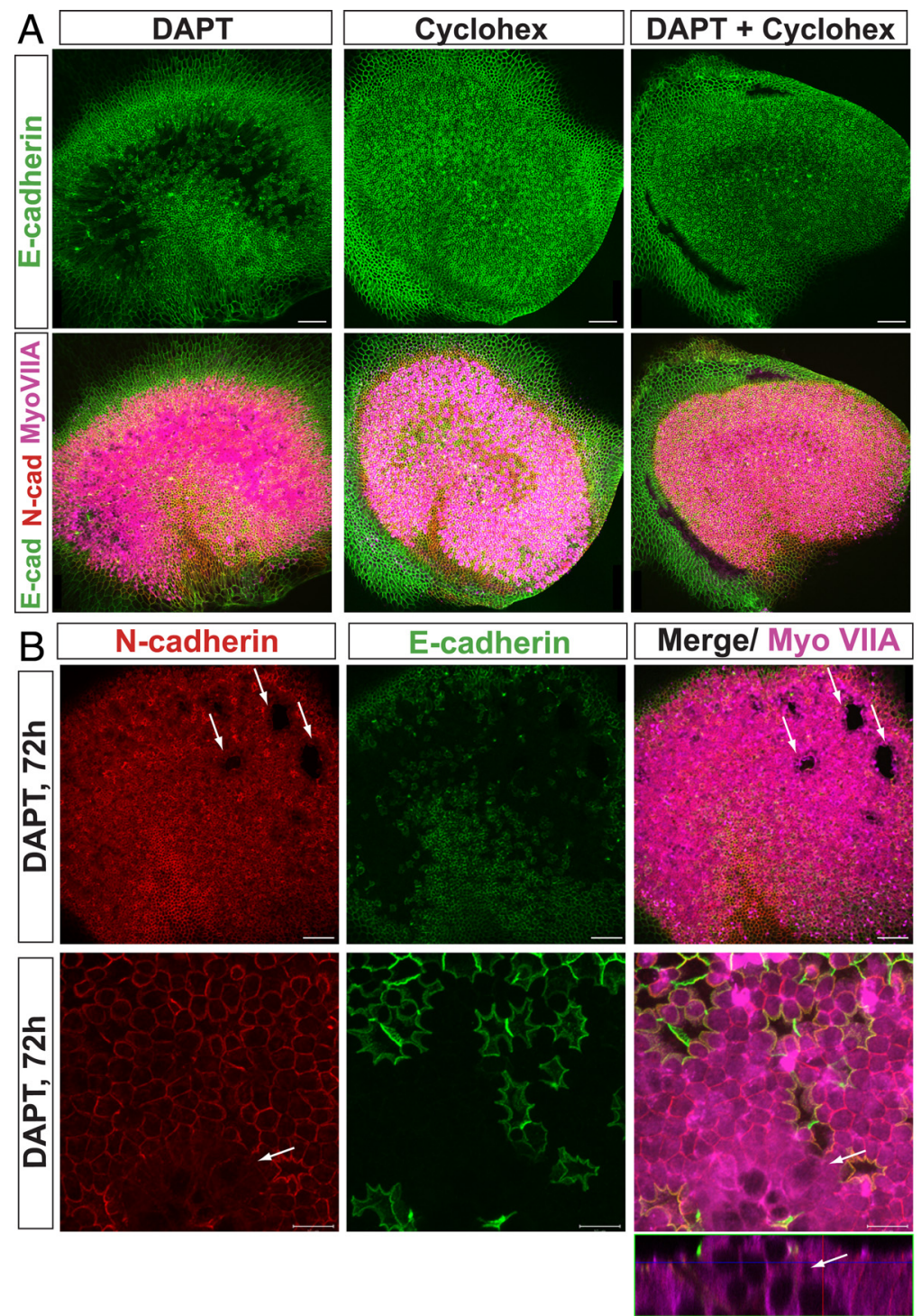

Figure 7. DAPT treatments progressively induce E-cadherin depletion and SC-to-HC conversion in a protein synthesis-dependent manner. $\boldsymbol{A}$, Whole-mount utricles treated with DAPT (50 $\mu \mathrm{m}$ ), DAPT and cycloheximide (40 $\mu \mathrm{m})$, or cycloheximide for $30 \mathrm{~h}$ and immunostained for N-cadherin (red), E-cadherin (green), and myosin VIIA (purple). B, Top, Images of whole-mount utricles showing conversion regions. Arrows point to "holes" in epithelium attributable to detachment of converted SCs from the basal lamina. Scale bars, $50 \mu \mathrm{m}$ Bottom, High-magnification images of the $x$ - $y$-axis and $z$-axis taken at the level of the striola, showing regions of conversion in which converting SCs resemble HCs and lift up from the basal lamina. Scale bars, $10 \mu \mathrm{m}$. A, Anterior; L, lateral.

region that is lateral to the line of hair bundle reversal are significantly wider than the AJRs of SC-SC junctions in the striola $(p<$ $0.05)$ (Fig. $8 B-E$ ). AJR widths decrease by $25 \%$ from the line of reversal to a point $20 \mu \mathrm{m}$ medial to it. These measurements show that thickening of the circumferential F-actin belts, which appears to be a unique age-related specialization of mammalian SCs (Burns et al., 2008), progresses faster in the extrastriolar SCs of neonatal mouse utricles than in the SCs in the striola. The increased E-cadherin and thicker F-actin belts observed at the junctions between the extrastriola SCs in neonates may indicate that extrastriolar SCs reach more advanced stages of cellular maturation than the striola SCs in the same utricles.

The vestibular SC phenotype becomes more stable as postnatal mammals age

Unlike the cochleae in embryonic and neonatal rodents, when adult rodent cochleae are subjected to $\gamma$-secretase inhibition, su- pernumerary HCs do not form (Hori et al., 2007). To determine whether differentiated vestibular SCs would exhibit age-dependent limitations in their capacity to change phenotype, we cultured utricles from mice that ranged from gestation day 18 (E18) to adulthood for $72 \mathrm{~h}$ in DAPT and DMSO media. Striolar SCs from E18, P2, P4, P8, P10, and P12 mice downregulated E-cadherin and converted into $\mathrm{HCs}$ in the presence of DAPT, but the regions in which SCs converted into HCs were much smaller in utricles from the $\mathrm{P} 8, \mathrm{P} 10$, and $\mathrm{P} 12$ mice than in utricles from the E18, P2, and P4 mice (Fig. 9) ( $n=3$ for each age), and the conversion took longer. In fact, the striola of the P12 utricles contained only a few, small and isolated regions of juxtaposed myosin-VIIA-positive, E-cadherin-negative cells (Fig. 9). No signs of E-cadherin depletion and no signs of phenotypic conversion were observed in utricles from P16 mice after culturing with DAPT for $72 \mathrm{~h}(n=5)$. None occurred in adult (P82) utricles after continuous culturing with DAPT for $72 \mathrm{~h}$ $(n=3), 5 \mathrm{~d}(n=4), 7 \mathrm{~d}(n=4)$, and $10 \mathrm{~d}$ $(n=4)$ (data not shown). Thus, inhibition of $\gamma$-secretase activity becomes progressively less effective at inducing SCs to convert to a HC phenotype as SCs mature during the first weeks of postnatal life.

\section{Discussion}

Our experiments indicate that E-cadherin is effectively restricted to SC-SC junctions in human and murine vestibular epithelia and show that age-related reductions in the phenotypic plasticity of SCs in rodents strongly correlate with the postnatal accumulation of E-cadherin in those junctions. When we cultured utricles from young mice with inhibitors of $\gamma$-secretase, large numbers of SCs in the striola internalized E-cadherin, expressed Atoh1, and progressively converted to a HC phenotype. Hes and Hey expression decreased in such GSI-treated utricles. However, scattered striolar SCs and the vast majority of the extrastriolar SCs maintained their junctional E-cadherin after GSI treatments. Such E-cadherin-expressing SCs exhibited no detectable changes in phenotype, but SC-to-HC phenotype conversion was pervasive in the cells that downregulated junctional E-cadherin. Morphometry showed that circumferential F-actin belts at the junctions between striolar SCs in young mice are significantly thinner than the belts in extrastriolar SCs lateral to the striola. Striolar E-cadherin depletion and SC-to-HC phenotype conversion declined with age, occurring in GSI-treated utricles from P12 mice but not in utricles from P16 and older mice. The findings here highlight the potential importance of junctional E-cadherin as a regulator of cellular phenotype stability. They also put differences in E-cadherin expression and differences in the repair capacities of hair cell epithelia in birds and mammals in a new context. 
Differences in E-cadherin expression and junction reinforcement are mirrored by differences in SC plasticity and $\mathrm{HC}$ regeneration

We found no notable differences in the localization of $\mathrm{N}$-cadherin in mammalian hair cell epithelia from what has been reported in birds (Warchol, 2007), but our findings show that E-cadherin expression differs significantly. The SC-specific expression and postnatal increases in E-cadherin that we measured here are strongly correlated with progressive thickening of the circumferential F-actin belts that bracket SC-SC junctions in mammals ( $r=0.94$ ) (Fig. 2) (Burns et al., 2008). Circumferential SC belt thickening inversely correlates, in turn, with postnatal declines in the propensity for SCs to change shape $(r=-0.99)$ and proliferate after damage to the murine HC epithelium $(r=$ -0.98) (Davies et al., 2007; Burns et al., 2008; Lu and Corwin, 2008). E-cadherin has been shown to influence cellular proliferation and differentiation in a number of other tissues and could limit SC plasticity either directly or indirectly (Takeichi, 1993; Gumbiner, 1996; Hines et al., 1999; Owens et al., 2000; Thiery and Sleeman, 2006). Although our results show that postnatal accumulation of E-cadherin and reinforcement of SC junctions correlate with declining SC plasticity in postnatal mammals, they do not reveal whether or how the specializations of mammalian SC-SC junctions may be causally related to lower plasticity.

\section{E-cadherin regulation in postnatal} mammalian utricles

Our results suggest that the initial postnatal increase in E-cadherin may be at least partially attributed to relief from transcriptional repression by the zinc-finger transcription factor Slug. Slug protein is present in the nucleus of young utricular SCs and is expressed at high levels during the first postnatal week, when Shug (Snai2) mRNA levels are tightly and inversely correlated with increasing E-cadherin mRNA (Fig. 2). In Madin-Darby canine kidney (MDCK) cells, Slug interacts with proximal E-boxes to fully repress E-cadherin transcription, and the expression of Slug is strongly correlated with loss of E-cadherin during epithelial-mesenchymal transitions in both MDCK cells and carcinomas (Hajra et al., 2002).

Postnatal accumulation of E-cadherin at SC-SC junctions also appears likely to derive in part from its stabilization via linkage to thick F-actin belts that become progressively more stable as the junctions between mammalian SCs mature (Burns et al., 2008; Cavey et al., 2008) (J. Burns and J. T. Corwin, unpublished
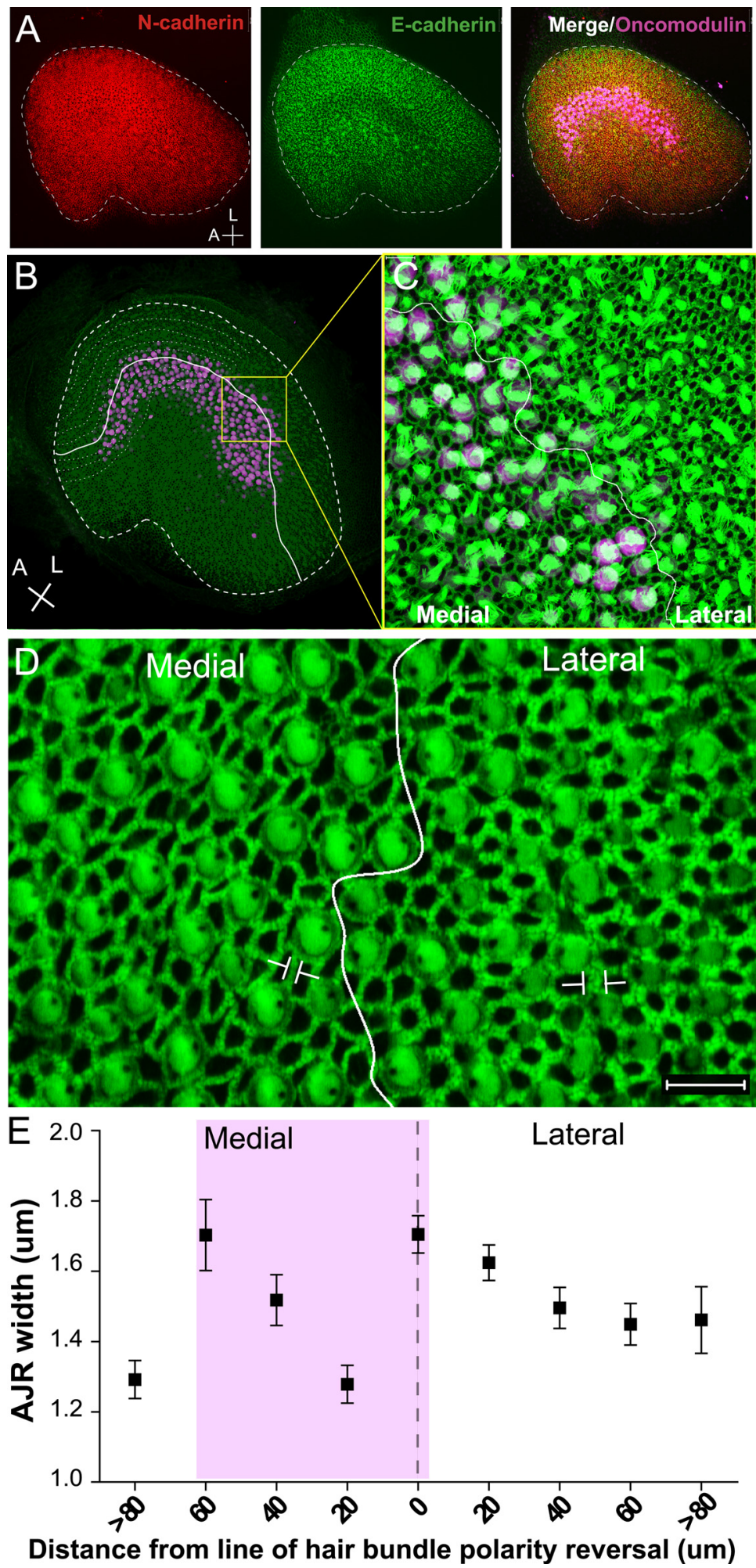

Figure 8. The width of circumferential $\mathrm{F}$-actin bands in P2 mice utricles is thinnest in striolar supporting cells. $A$, Image of a P2 mouse whole-mount utricle cultured with the vehicle for $48 \mathrm{~h}$ and labeled for $\mathrm{E}$-cadherin (green), $\mathrm{N}$-cadherin (red), and striolar marker oncomodulin (purple). Scale bars, $50 \mu \mathrm{m} . \boldsymbol{B}, \mathrm{P} 2$ mouse utricle labeled for F-actin (green) and oncomodulin (purple). The solid white line marks the line of hair bundle polarity reversal, and the thick dashed line marks the outer edge of the sensory epithelium. The thinner dashed lines illustrate transects followed in the quantification process, in which the widths of $100 \mathrm{AJRs}$ along each side of the line of polarity reversal were measured in relation to the shortest distance from the measured cell to the line of reversal. $\boldsymbol{C}$, Higher-magnification image of $\boldsymbol{B}$. $\boldsymbol{D}$, High-resolution image showing the difference in widths of $\mathrm{F}$-actin bands in the regions lateral and medial to the line of polarity reversal (white line) at the level of the striola. $\boldsymbol{E}$, Quantification of AJR widths plotted as a function of the shortest distance to the line of hair bundle polarity reversal. Data were binned in $20 \mu \mathrm{m}$ intervals. Data point 0 and dashed line indicate the line of reversal. Purple shadowing represents the location of the striola. 

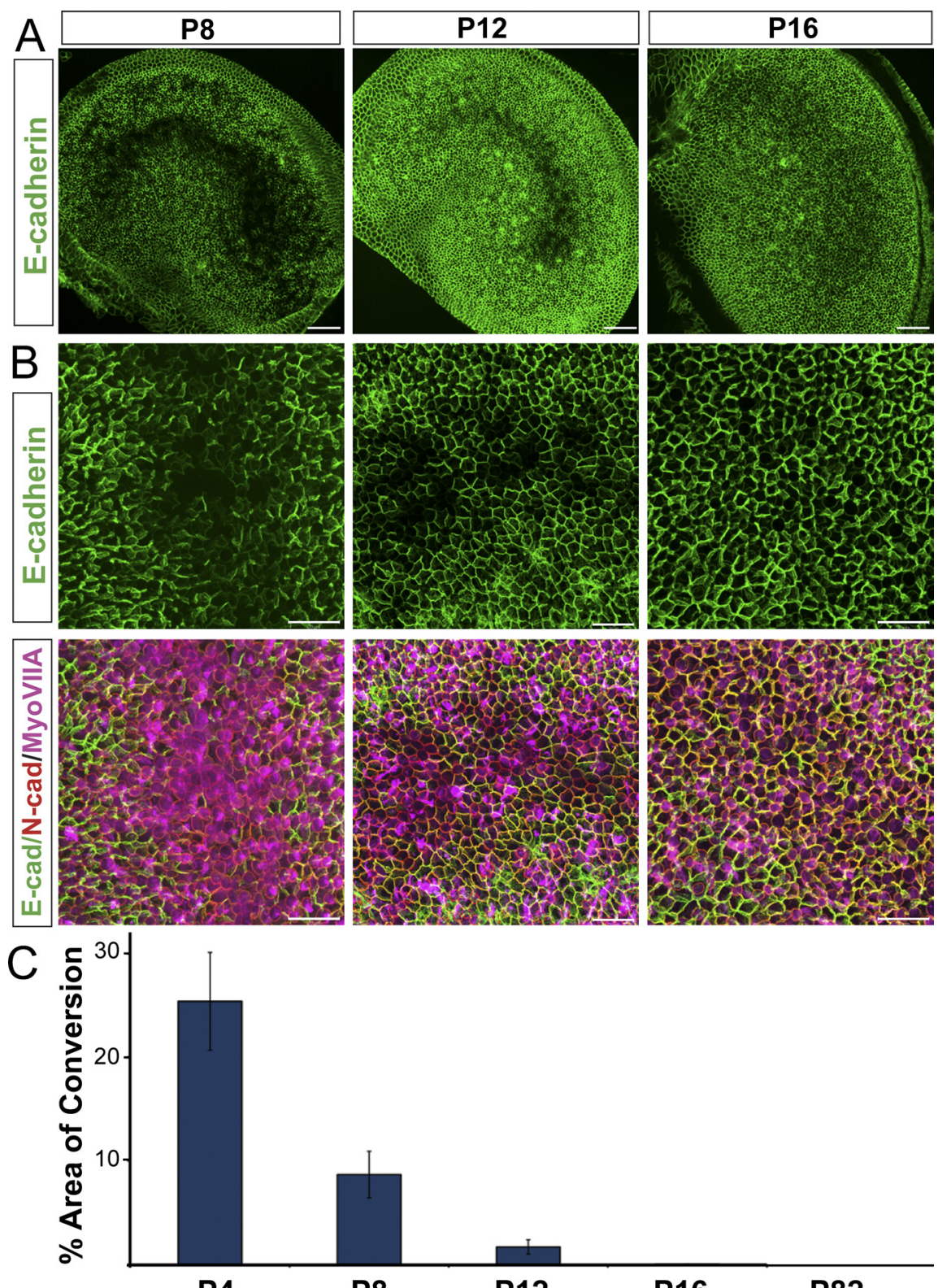

P4

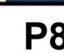

P8

\section{P12}

\section{P16}

Figure 9. The number of SCs that downregulate E-cadherin and convert into $\mathrm{HCS}$ after DAPT treatments declines with postnatal maturation, so that by P16 SCs no longer convert into HCs. Whole mount utricles harvested from P8, P12 and P16, treated with DAPT for $72 \mathrm{~h}$ and labeled for E-cadherin (green), N-cadherin (red) and myosin VIIA (purple). A, Images of whole utricles showing regions of E-cadherin depletion. Scale bars are $50 \mu \mathrm{m}$. Please note that E-cadherin levels are not comparable between the different age group images in this figure. Image intensity was individually optimized for each age. Although E-cadherin levels appear to be lower in the striolar regions at all ages, E-cadherin is completely absent only in the P8 and P12 shown. B, Higher magnification images taken at the level of the striola, showing that the regions of conversion become progressively smaller until disappearing at P16. Scale bars are $10 \mu \mathrm{m}$. C, Quantification of area exhibiting SC-to-HC conversion as a function of postnatal age $(n=4$ for P4 and $\mathrm{P} 8, n=5$ for $\mathrm{P} 12, n=3$ for $\mathrm{P} 16$ and $n=3$ for P82).

observations). E-cadherin, in turn, is likely to anchor and stabilize the SC cytoskeleton, as it does in other cells (Chausovsky et al., 2000; Waterman-Storer et al., 2000), thus contributing to positive feedback interactions, which ultimately may stabilize cellular phenotypes.

The lack of evidence for GSI-induced transcriptional regulation (Fig. 5 ), together with the rapid and complete removal of membrane E-cadherin and the transient appearance of E-cadherin immunopositive cytoplasmic puncta that follow GSI treatments (Fig. 6), point to regulation at the level of E-cadherin endocytosis. Such internalization may be specific to E-cadherin, because $\mathrm{N}$-cadherin at those junctions appeared unaffected. E-cadherin depletion is not attributable to shed-

\section{P82}

ding of a cleaved intracellular domain, which is known to be dependent on $\gamma$-secretase activity that is blocked by GSIs (Fortini, 2002).

The 15-18 h lag between the GSI exposure and the internalization of E-cadherin led us to test and confirm that the GSI-induced internalization is dependent on protein synthesis (Fig. 7), as would be the case if E-cadherin internalization were downstream of Atoh1 induction. E-cadherin internalization may influence signaling through the release of $\beta$-catenin, which has been shown to activate Atoh1 transcription in the ear (Shi et al., 2010), but signaling in the opposite direction has not been established.

\section{Maintenance of the SC phenotype in the young mice requires continuous \\ Notch signaling}

In embryonic ears, cell fate determination is mediated through surface ligands expressed by nascent HCs. Those ligands bind to Notch receptors of neighboring cells, which inhibits those cells from adopting the default HC phenotype and causes them to develop as SCs (Kelley, 2006). When Notch signaling has been interrupted during development of the ear by GSI treatments and by genetic deletions of Notch ligands and the RBPJ (CBF1) genes, overproduction of HCs results (Lanford et al., 1999; Kiernan et al., 2005; Brooker et al., 2006; Yamamoto et al., 2006). Surprisingly, our experiments show that ongoing activity in the Notch pathway is required well into the second postnatal week for the maintenance of the SC phenotype in the striola (Figs. 3-5).

The difference in the response of striolar and extrastriolar SCs to GSI treatment (Figs. $3,4,7)$ suggests that Notch signaling alone does not control the maintenance of the SC phenotype in young mice. One possibility is that Notch signaling is not active in the extrastriolar regions of postnatal utricles. It has been reported that the striola is the predominant site of Hes5 expression in rodent utricles at late embryonic stages, whereas Hes 1 is expressed throughout the utricle (Zheng et al., 2000). Such differential expression of Notch pathway components may contribute to regional differences in the requirement for Notch signaling postnatally. Another explanation for the predominance of SC-to-HC conversion in the striola may be related to the higher levels of membrane E-cadherin and thicker circumferential F-actin belts that are present in extrastriolar SCs of young neonatal utricles (Fig. 8). The results suggest that the extrastriolar SCs in neonates may reach an advanced state of maturity before the SCs in the striola.

Increased E-cadherin may help maintain phenotypic stability of mature SCs

Although a decrease in the dependence on Notch activity may explain why even striolar SCs become unresponsive to GSI treat- 
ments with age (Fig. 9), the results suggest that the unique junctional changes that occur in maturing mammalian SCs may help to confer persistent phenotypic stability. The decline in the capacity of SCs to convert to a sensory receptor phenotype occurs in parallel with the accumulations of E-cadherin in the membranes and F-actin in the circumferential belts at the junctions of postnatally maturing SCs. Both processes of accumulation occur more quickly in extrastriolar SCs than in striolar SCs, and both become considerably more pronounced in all SCs after 2 weeks of life. In addition, our experimental findings show that SCs do not undergo SC-to-HC phenotypic conversion in the absence of GSI-induced internalization of E-cadherin. Therefore, it appears possible and perhaps likely that the postnatal accumulation and increased stability of junctional E-cadherin that has been described here is linked to the growth of uniquely reinforced, circumferential F-actin belts, which together contribute to SC phenotypic stability and help to limit the capacity of the mammalian ear for regeneration.

\section{References}

Bermingham NA, Hassan BA, Price SD, Vollrath MA, Ben-Arie N, Eatock RA, Bellen HJ, Lysakowski A, Zoghbi HY (1999) Math1: an essential gene for the generation of inner ear hair cells. Science 284:1837-1841.

Brooker R, Hozumi K, Lewis J (2006) Notch ligands with contrasting functions: Jagged 1 and Delta1 in the mouse inner ear. Development 133:1277-1286.

Burns J, Christophel JJ, Collado MS, Magnus C, Carfrae M, Corwin JT (2008) Reinforcement of cell junctions correlates with the absence of hair cell regeneration in mammals and its occurrence in birds. J Comp Neurol 511:396-414.

Cavey M, Rauzi M, Lenne PF, Lecuit T (2008) A two-tiered mechanism for stabilization and immobilization of E-cadherin. Nature 453:751-756.

Chausovsky A, Bershadsky AD, Borisy GG (2000) Cadherin-mediated regulation of microtubule dynamics. Nat Cell Biol 2:797-804.

Davies D, Magnus C, Corwin JT (2007) Developmental changes in cellextracellular matrix interactions limit proliferation in the mammalian inner ear. Eur J Neurosci 25:985-998.

Davies S, Forge A (1987) Preparation of the mammalian organ of Corti for scanning electron microscopy. J Microsc 147:89-101.

Denman-Johnson K, Forge A (1999) Establishment of hair bundle polarity and orientation in the developing vestibular system of the mouse. J Neurocytol 28:821-835.

Doetzlhofer A, Basch ML, Ohyama T, Gessler M, Groves AK, Segil N (2009) Hey2 regulation by FGF provides a Notch-independent mechanism for maintaining pillar cell fate in the organ of Corti. Dev Cell 16:58-69.

Favre D, Bagger-Sjöbäck D, Mbiene JP, Sans A (1986) Freeze-fracture study of the vestibular hair cell surface during development. Anat Embryol (Berl) 175:69-76.

Forge A, Souter M, Denman-Johnson K (1997) Structural development of sensory cells in the ear. Semin Cell Dev Biol 8:225-237.

Fortini ME (2002) Gamma-secretase-mediated proteolysis in cell-surfacereceptor signalling. Nat Rev Mol Cell Biol 3:673-684.

Gu R, Montcouquiol M, Marchionni M, Corwin JT (2007) Proliferative responses to growth factors decline rapidly during postnatal maturation of mammalian hair cell epithelia. Eur J Neurosci 25:1363-1372.

Gubbels SP, Woessner DW, Mitchell JC, Ricci AJ, Brigande JV (2008) Functional auditory hair cells produced in the mammalian cochlea by in utero gene transfer. Nature 455:537-541.

Gumbiner BM (1996) Cell adhesion: the molecular basis of tissue architecture and morphogenesis. Cell 84:345-357.

Hackett L, Davies D, Helyer R, Kennedy H, Kros C, Lawlor P, Rivolta MN, Holley M (2002) E-cadherin and the differentiation of mammalian vestibular hair cells. Exp Cell Res 278:19-30.

Hajra KM, Chen DY, Fearon ER (2002) The SLUG zinc-finger protein represses E-cadherin in breast cancer. Cancer Res 62:1613-1618.

Hayashi T, Kokubo H, Hartman BH, Ray CA, Reh TA, BerminghamMcDonogh O (2008) Hesr1 and Hesr2 may act as early effectors of Notch signaling in the developing cochlea. Dev Biol 316:87-99.

Hines MD, Jin HC, Wheelock MJ, Jensen PJ (1999) Inhibition of cadherin function differentially affects markers of terminal differentiation in cultured human keratinocytes. J Cell Sci 112:4569-4579.

Hori R, Nakagawa T, Sakamoto T, Matsuoka Y, Takebayashi S, Ito J (2007)
Pharmacological inhibition of Notch signaling in the mature guinea pig cochlea. Neuroreport 18:1911-1914.

Kelley MW (2006) Regulation of cell fate in the sensory epithelia of the inner ear. Nat Rev Neurosci 7:837-849.

Kiernan AE, Cordes R, Kopan R, Gossler A, Gridley T (2005) The Notch ligands DLL1 and JAG2 act synergistically to regulate hair cell development in the mammalian inner ear. Development 132:4353-4362.

Kim TS, Nakagawa T, Endo T, Iguchi F, Murai N, Naito Y, Ito J (2002) Alteration of E-cadherin and beta-catenin in mouse vestibular epithelia during induction of apoptosis. Neurosci Lett 329:173-176.

Kim TS, Nakagawa T, Kitajiri S, Endo T, Takebayashi S, Iguchi F, Kita T, Tamura T, Ito J (2005) Disruption and restoration of cell-cell junctions in mouse vestibular epithelia following aminoglycoside treatment. Hear Res 205:201-209.

Lanford PJ, Lan Y, Jiang R, Lindsell C, Weinmaster G, Gridley T, Kelley MW (1999) Notch signalling pathway mediates hair cell development in mammalian cochlea. Nat Genet 21:289-292.

Li A, Xue J, Peterson EH (2008) Architecture of the mouse utricle: macular organization and hair bundle heights. J Neurophysiol 99:718-733.

Lu Z, Corwin JT (2008) The influence of glycogen synthase kinase 3 in limiting cell addition in the mammalian ear. Dev Neurobiol 68:1059-1075.

Lumpkin EA, Collisson T, Parab P, Omer-Abdalla A, Haeberle H, Chen P, Doetzlhofer A, White P, Groves A, Segil N, Johnson JE (2003) Math1driven GFP expression in the developing nervous system of transgenic mice. Gene Expr Patterns 3:389-395.

Mbiene JP, Favre D, Sans A (1984) The pattern of ciliary development in fetal mouse vestibular receptors. A qualitative and quantitative SEM study. Anat Embryol (Berl) 170:229-238.

Meyers JR, Corwin JT (2007) Shape change controls supporting cell proliferation in lesioned mammalian balance epithelium. J Neurosci 27:4313-4325.

Montcouquiol M, Corwin JT (2001) Intracellular signals that control cell proliferation in mammalian balance epithelia: key roles for phosphatidylinositol-3 kinase, mammalian target of rapamycin, and S6 kinases in preference to calcium, protein kinase $\mathrm{C}$, and mitogen-activated protein kinase. J Neurosci 21:570-580.

Owens DW, Brunton VG, Parkinson EK, Frame MC (2000) E-cadherin at the cell periphery is a determinant of keratinocyte differentiation in vitro. Biochem Biophys Res Commun 269:369-376.

Shi F, Cheng YF, Wang XL, Edge AS (2010) Beta-catenin up-regulates Atoh1 expression in neural progenitor cells by interaction with an Atoh1 3' enhancer. J Biol Chem 285:392-400.

Simmons DD, Tong B, Schrader AD, Hawkes AJ (2010) Oncomodulin identifies different hair cell types in the mammalian inner ear. J Comp Neurol 518:3785-3802.

Takebayashi S, Yamamoto N, Yabe D, Fukuda H, Kojima K, Ito J, Honjo T (2007) Multiple roles of Notch signaling in cochlear development. Dev Biol 307:165-178.

Takeichi M (1993) Cadherins in cancer: implications for invasion and metastasis. Curr Opin Cell Biol 5:806-811.

Thiery JP, Sleeman JP (2006) Complex networks orchestrate epithelialmesenchymal transitions. Nat Rev Mol Cell Biol 7:131-142.

Warchol ME (2007) Characterization of supporting cell phenotype in the avian inner ear: implications for sensory regeneration. Hear Res 227:11-18.

Waterman-Storer CM, Salmon WC, Salmon ED (2000) Feedback interactions between cell-cell adherens junctions and cytoskeletal dynamics in newt lung epithelial cells. Mol Biol Cell 11:2471-2483.

Woods C, Montcouquiol M, Kelley MW (2004) Math1 regulates development of the sensory epithelium in the mammalian cochlea. Nat Neurosci $7: 1310-1318$.

Yamamoto N, Tanigaki K, Tsuji M, Yabe D, Ito J, Honjo T (2006) Inhibition of Notch/RBP-J signaling induces hair cell formation in neonate mouse cochleas. J Mol Med 84:37-45.

Zhao S, Fernald RD (2005) Comprehensive algorithm for quantitative realtime polymerase chain reaction. J Comput Biol 12:1047-1064.

Zhao Y, Yamoah EN, Gillespie PG (1996) Regeneration of broken tip links and restoration of mechanical transduction in hair cells. Proc Natl Acad Sci U S A 93:15469-15474.

Zheng JL, Gao WQ (2000) Overexpression of Math1 induces robust production of extra hair cells in postnatal rat inner ears. Nat Neurosci 3:580-586.

Zheng JL, Shou J, Guillemot F, Kageyama R, Gao WQ (2000) Hes1 is a negative regulator of inner ear hair cell differentiation. Development 127: $4551-4560$. 